\title{
Delayed diagnosis of pulmonary embolism
}

\author{
Chia-Jung Hsieh ${ }^{1}$, Meng-Jung Chen ${ }^{2 *}$ \\ ${ }^{1}$ Department of Medical Technology, Chung-Hwa University of Medical Technology, Tainan, Chinese Taipei \\ ${ }^{2}$ Department of Emergency Medicine, Chi-Mei Medical Center, Tainan, Chinese Taipei \\ Email: ${ }^{\text {ericmjc@yahoo.com.tw }}$
}

Received 2 August 2012; revised 22 September 2012; accepted 20 October 2012

\begin{abstract}
Pulmonary embolism is a common and potentially life threatening condition. Delays in pulmonary embolism diagnosis represent an important problem. It is crucial to have early diagnosis of pulmonary embolism if one wishes to reduce its complication. We retrospecttively reviewed our patients who visited our emergency department with a final diagnosis of pulmonary embolism. We found that delayed diagnosis of pulmonary embolism usually happened when patients did not present with the classical symptoms and signs leading to other medical diagnosis at first. Clinical signs and symptoms for pulmonary embolism are mostly nonspecific; therefore, patients suspected of having pulmonary embolism - because of unexplained chest pain, shortness of breath or tachycardia with or without the risk factors for pulmonary embolism-must undergo diagnostic tests including D-dimer or CT pulmonary angiography if indicated until the diagnosis is ruled out.
\end{abstract}

Keywords: Delayed Diagnosis; Emergency Department; Pulmonary Embolism

\section{INTRODUCTION}

Pulmonary embolism is a blockage of one of the arteries of lung which cause obstruction of blood flow to the lung and high pressure on the right ventricle leading to symptoms and signs such as shortness of breath, chest pain and rapid heart rate [1]. Blood clots are usually from leg veins where blood flows poorly [2]. This pooling of blood and platelet leads to clot formation. When the clot blocks the pulmonary artery, it is not easy for heart to pump blood through the lungs. The lungs react by releasing chemicals that may cause the blood vessels to spasm, leading further difficulty for the heart to pump. These factors contribute to shortness of breath and chest pains. Pulmonary embolism can not be easily diagnosed since many symptoms mimic other cardiopulmonary dis-

${ }^{*}$ Corresponding author. eases [3]. Diagnosis is made under high clinical suspicion with appropriate laboratory tests and image study. Treatment requires anticoagulant and thrombolytic or surgical intervention in severe cases. Pulmonary embolism is a common and life threatening condition. Delays in pulmonary embolism diagnosis represent an important problem [4]. Massive pulmonary embolism is only next to sudden cardiac death as a cause of sudden death [5]. It is crucial to have early diagnosis of pulmonary embolism if one wishes to reduce its mortality [6].

\section{MATERIAL AND METHODS}

We retrospectively reviewed our patients who visited our emergency department with a final diagnosis of pulmonary embolism. Early diagnosis was defined as diagnosis less than 12 hours from the arrival at emergency department, and delayed diagnosis as more than 12 hours after arrival at the emergency department. Analysis involved history, symptoms, signs and diagnostic tests used.

\section{RESULTS}

The median time from arrival at emergency department to diagnosis of pulmonary embolism was 18 hours, and $50 \%$ of patients had delayed diagnosis. Even in the early diagnosis group, the average time of diagnosis is 9 hours which indicate that pulmonary embolism is often not recognized in the beginning. Delayed diagnoses are frequently made on the second or third emergency department visit or later on after admission to ward. Patients who received a first impression such as pleurisies, hyperventilation, coronary artery disease or pneumonia had longer times from the arrival at emergency department to diagnosis of pulmonary embolism, whereas patients with immobility and other risk factors such as recent surgery and deep vein thrombosis (DVT) or classic symptoms and signs such as shortness of breath and chest pain had shorter times to diagnosis of pulmonary embolism. Early diagnosis of pulmonary embolism can be better achieved if diagnostic tests including D-dimer are used in the beginning with CT pulmonary angiography afterwards. 
Table 1. Variables associated with delayed diagnosis.

\begin{tabular}{lcc}
\hline Variables & Early diagnosis $<12$ hours & Delayed diagnosis $>12$ hours \\
\hline Patients numbers & 20 & 20 \\
Shortness of breath percentage & 75 & 25 \\
Chest pain percentage & 60 & 35 \\
DVT percentage & 20 & 5 \\
D-dimer used percentage & 60 & 10 \\
CT scan percentage & 85 & 5 \\
Recent surgery percentage & 25 & 5 \\
Average time of diagnosis (hours) & 9 & 27 \\
\hline
\end{tabular}

\section{DISCUSSION}

Despite diagnostic advances, delays in pulmonary embolism diagnosis are common and represent an important problem. In patients with pulmonary embolism, mortality can be prevented with early diagnosis and therapy [7]. Unfortunately, the diagnosis is frequently delayed because patients with pulmonary embolism usually have nonspecific signs and symptoms. The lungs are usually normal on physical examination. The variability of presentation of pulmonary embolism leads to missing the diagnosis easily [8]. The challenge is that the presentation with sudden onset of pleurisy, shortness of breath, and hypoxia all together is not common at all. Patents frequently had no risk factors like drug, hormone use, tumor, genetic thrombophilia (protein $\mathrm{C}$ deficiency, protein $\mathrm{S}$ deficiency, antithrombin deficiency, hyperhomocysteinemia and plasminogen/fibrinolysis disorders) or acquired thrombophilia (antiphospholipid syndrome, nephrotic syndrome, paroxysmal nocturnal hemoglobinuria) [9].

Further, routine laboratory findings may be nonspecific and not helpful in pulmonary embolism including chest $\mathrm{X}$ ray and electrocardiogram, however these tests may suggest another diagnosis leading to admission for further workup and subsequent correct diagnosis [10]. The under use of D-dimer may also lead to delayed diagnosis of pulmonary embolism. Although D-dimer is not a specific examination, its good sensitivity and negative likelihood ratio contribute to differential diagnosis leading further to more appropriate tests. While pulmonary angiography is the standard diagnosis of pulmonary embolism, it is frequently replaced by CT pulmonary angiography because of its accessibility with improved sensitivity and specificity in diagnosing pulmonary embolism. We usually perform the D-dimer test first when pulmonary embolism is suspected. If the D-dimer test is negative, the likelihood of pulmonary embolism is low and CT pulmonary embolism may not be performed. CT pulmonary embolism is usually performed if D-dimer is elevated. However D-dimer is a nonspecific test, so only a certain percentage will show pulmonary embolism and the rest may reveal some other diagnoses. The clinical scoring system adding other risk factors such as immobilization, deep vein thrombosis, tachycardia, hemoptysis and malignancy could be also of help [11].

The limitation of this study is the small numbers of patients included in only one medical center. However other study in the literature showed a significant numbers of patients who died unexpectedly of pulmonary embolism had complained of some symptoms and signs, often for weeks, before dying. Forty percent of these patients had been seen by more than one physician prior to their death. This indicates that delayed diagnosis of pulmonary embolism is a worldwide problem which needed to be taken seriously [12].

\section{CONCLUSION}

Clinical signs and symptoms for pulmonary embolism are nonspecific; therefore, patients suspected of having pulmonary embolism - because of unexplained chest pain, shortness of breath or tachycardia with or without the risk factors for pulmonary embolism-must undergo diagnostic tests including D-dimer or CT pulmonary angiography if indicated until the diagnosis is ruled out or an alternative diagnosis is confirmed.

\section{REFERENCES}

[1] Stein, P.D., Beemath, A., Matta, F., Weg, J.G., Yusen, R.D., Hales, C.A., et al. (2007) Clinical characteristics of patients with acute pulmonary embolism: Data from PIOPED II. The American Journal of Medicine, 120, 871879. doi:10.1016/j.amjmed.2007.03.024 
[2] Kline, J.A. and Runyon, M.S. (2006) Pulmonary embolism and deep venous thrombosis. In: Marx, J.A., Hockenberger, R.S. and Walls, R.M., Eds., Rosen's Emergency Medicine Concepts and Clinical Practice, 2, 13681382.

[3] Ozsu, S., Oztuna, F., Bulbul, Y., et al. (2011) The role of risk factors in delayed diagnosis of pulmonary embolism. American Journal of Emergency Medicine, 29, 26-32. doi:10.1016/j.ajem.2009.07.005

[4] Biss, T.T., Brandão, L.R., Kahr, W.H., Chan, A.K. and Williams, S. (2008) Clinical features and outcome of pulmonary embolism in children. British Journal of Haematology, 142, 808-818.

doi:10.1111/j.1365-2141.2008.07243.x

[5] Tapson, V.F. (2008) Acute pulmonary embolism. The New England Journal of Medicine, 358, 1037-1052. doi:10.1056/NEJMra072753

[6] Sandler, D.A. and Martin, J.F. (1989) Autopsy proven pulmonary embolism in hospital patients: Are we detecting enough deep vein thrombosis? Journal of the Royal Society of Medicine, 82, 203-205.

[7] Meyer, G., Planquette, B. and Sanchez, O. (2008) Longterm outcome of pulmonary embolism. Current Opinion in Hematology, 15, 499-503. doi:10.1097/MOH.0b013e3283063a51

[8] Garcia, D., Ageno, W. and Libby, E. (2005) Update on the diagnosis and management of pulmonary embolism. British Journal of Haematology, 131, 301-312. doi:10.1111/j.1365-2141.2005.05752.x

[9] Konstantinides, S. (2008) Clinical practice. Acute pulmonary embolism. The New England Journal of Medicine, 359, 2804-2813. doi:10.1056/NEJMcp0804570

[10] Douma, R.A., Mos, I.C., Erkens, P.M., Nizet, T.A., Durian, M.F., Hovens, M.M., et al. (2011) Performance of 4 clinical decision rules in the diagnostic management of acute pulmonary embolism: A prospective cohort study. Annals of Internal Medicine, 154, 709-718.

[11] Wells, P.S., Ginsberg, J.S., Anderson, D.R., Kearon, C., Gent, M., Turpie, A.G., et al. (1998) Use of a clinical model for safe management of patients with suspected pulmonary embolism. Annals of Internal Medicine, 129, 997-1005.

[12] Smith, S.B., Geske, J.B. and Morgenthaler, T.I. (2012) Risk factors associated with delayed diagnosis of acute pulmonary embolism. The Journal of Emergency Medicine, 42, 1-6. doi:10.1016/j.jemermed.2011.06.004 